\title{
Investigations on the Theory of Riemann Zeta Function I: New Functional Equation, Integral Representation and Laurent Expansion for Riemann's Zeta Function
}

\author{
Edigles Guedes ${ }^{1}$, Prof. Dr. Raja Rama Gandhi ${ }^{2}$ and Srinivas Kishan Anapu ${ }^{3}$ \\ ${ }^{1}$ Number Theorist, Pernambuco, Brazil. \\ ${ }^{2}$ Resource person in Math for Oxford University Press, Professor in Math, BITS-Vizag. \\ ${ }^{3}$ CEO, Voice of Bigdata, Austin, TX.
}

ABSTRACT. We developed a new functional equation and a new integral representation for the Riemann zeta function.

\section{INTRODUCTION}

Our main goal is the development of these formulas:

$$
\begin{gathered}
\left(2^{1-s}-1\right) \Gamma\left(\frac{s}{2}\right) \pi^{-s+1 / 2} \zeta(s)=\Gamma\left(\frac{1-s}{2}\right)\left[2^{1-s}+\zeta\left(1-s, \frac{3}{2}\right)\right] \\
\zeta(\mathrm{s})=\frac{2^{\mathrm{s}}}{2^{\mathrm{s}-1}}+\frac{3^{1-\mathrm{s}}}{\left(2-2^{1-\mathrm{s}}\right)(\mathrm{s}-1)}+\frac{2^{\mathrm{s}-1}}{3^{\mathrm{s}}\left(2^{\mathrm{s}}-1\right)}+\frac{2}{2^{\mathrm{s}}-1} \int_{0}^{\infty} \frac{\sin \left[\mathrm{stan}^{-1}\left(\frac{2 \mathrm{t}}{3}\right)\right]}{\left[\left(\frac{3}{2}\right)^{2}+\mathrm{t}^{2}\right]^{\mathrm{s} / 2}} \frac{\mathrm{dt}}{\mathrm{e}^{2 \pi \mathrm{t}}-1}
\end{gathered}
$$

and

$$
\zeta(s)=\frac{2^{s}}{2^{s}-1}+\frac{1}{(s-1)\left(2^{s}-1\right)}+\frac{1}{2^{s}-1} \sum_{n=0}^{\infty} \frac{(-1)^{n}}{n !} \gamma_{n}\left(\frac{3}{2}\right)(s-1)^{n} .
$$

which converges more rapidly than the known Laurent expansion [see 5]:

$$
\zeta(s)=\frac{1}{s-1}+\sum_{n=0}^{\infty} \frac{(-1)^{n}}{n !} \gamma_{n}(1)(s-1)^{n} .
$$

\section{LEMMAS AND THEOREMS}

LEMMA 1. For $n \in \mathbb{Z}_{\geq 1}$ and $\alpha \in \mathbb{R}-\{0,1\}$, then

$$
\frac{1}{n}=\frac{\alpha}{(\alpha-1) n}-\frac{\alpha}{\alpha(\alpha-1) n}
$$

Proof. We expand the right-hand side of (2.1)

$$
\begin{aligned}
\frac{\alpha}{(\alpha-1) n}-\frac{\alpha}{\alpha(\alpha-1) n} & =\frac{\alpha}{\alpha-1}\left(\frac{1}{n}-\frac{1}{\alpha n}\right) \\
& =\frac{\alpha}{(\alpha-1)}\left(\frac{\alpha-1}{\alpha n}\right)=\frac{1}{n} . \square
\end{aligned}
$$

LEMMA 2. For $n \in \mathbb{Z}_{\geq 1}$ and $m \in \mathbb{Z}_{>1}$, then

$$
\frac{1}{n}=\frac{1}{n-1}-\frac{1}{(n-1) n} .
$$

Proof. Let $\alpha=n$, in Lemma 1. 
THEOREM 1. Let $\operatorname{Re}(s)>0$ and $s \neq 1$, then

$$
\zeta(s)=\frac{2^{s}}{2^{s}-1}+\frac{\zeta\left(s, \frac{3}{2}\right)}{2^{s}-1}
$$

where $\zeta(s)$ is the Riemann zeta function and $\zeta(s, a)$ is the Hurwitz zeta function.

Proof. For $\operatorname{Re}(s)>0$, then [see 1]

$$
\zeta(s)=\frac{1}{1-2^{1-s}} \sum_{n=1}^{\infty} \frac{(-1)^{n-1}}{n^{s}} \Rightarrow \zeta(s)-\frac{1}{1-2^{1-s}}=\frac{1}{1-2^{1-s}} \sum_{n=2}^{\infty} \frac{(-1)^{n-1}}{n^{s-1}} \cdot \frac{1}{n} .
$$

Substituting (2.2) in (2.4), we obtain

$$
\begin{gathered}
\zeta(s)-\frac{1}{1-2^{1-s}}=\frac{1}{1-2^{1-s}} \sum_{n=2}^{\infty} \frac{(-1)^{n-1}}{(n-1) n^{s-1}}-\frac{1}{1-2^{1-s}} \sum_{n=2}^{\infty} \frac{(-1)^{n-1}}{(n-1) n^{s}}, \\
\zeta(s)+\frac{1}{1-2^{1-s}} \sum_{n=2}^{\infty} \frac{(-1)^{n-1}}{(n-1) n^{s}}=\frac{1}{1-2^{1-s}}+\frac{1}{1-2^{1-s}} \sum_{n=2}^{\infty} \frac{(-1)^{n-1}}{(n-1) n^{s-1}} .
\end{gathered}
$$

On the other hand, in [2, p. 9], we encounter

$$
\int_{0}^{\infty} e^{-n x} x^{s-1} d x=\frac{\Gamma(s)}{n^{s}}
$$

for $\operatorname{Re}(s)>0$ and $n>0$.

We set (2.6) in (2.5)

$$
\begin{aligned}
& \zeta(s)+\frac{1}{\left(1-2^{1-s}\right) \Gamma(s)} \sum_{n=2}^{\infty} \frac{(-1)^{n-1}}{n-1} \int_{0}^{\infty} e^{-n x} x^{s-1} d x \\
& =\frac{1}{1-2^{1-s}}+\frac{1}{\left(1-2^{1-s}\right) \Gamma(s)} \sum_{n=2}^{\infty} \frac{(-1)^{n-1} n}{n-1} \int_{0}^{\infty} e^{-n x} x^{s-1} d x, \\
& \zeta(s)+\frac{1}{\left(1-2^{1-s}\right) \Gamma(s)} \int_{0}^{\infty}\left(\sum_{n=2}^{\infty} \frac{(-1)^{n-1}}{n-1} e^{-n x}\right) x^{s-1} d x \\
& =\frac{1}{1-2^{1-s}}+\frac{1}{\left(1-2^{1-s}\right) \Gamma(s)} \int_{0}^{\infty}\left(\sum_{n=2}^{\infty} \frac{(-1)^{n-1} n}{n-1} e^{-n x}\right) x^{s-1} d x, \\
& \zeta(s)-\frac{1}{\left(1-2^{1-s}\right) \Gamma(s)} \int_{0}^{\infty} \log \left(e^{-x}+1\right) e^{-x} x^{s-1} d x \\
& =\frac{1}{1-2^{1-s}}-\frac{1}{\left(1-2^{1-s}\right) \Gamma(s)} \int_{0}^{\infty}\left[\frac{\left(e^{-x}+1\right) \log \left(e^{-x}+1\right)+e^{-x}}{e^{x}+1}\right] x^{s-1} d x, \\
& \zeta(s)-\frac{1}{\left(1-2^{1-s}\right) \Gamma(s)} \int_{0}^{\infty} \log \left(e^{-x}+1\right) e^{-x} x^{s-1} d x \\
& =\frac{1}{1-2^{1-s}}-\frac{1}{\left(1-2^{1-s}\right) \Gamma(s)} \int_{0}^{\infty}\left[\frac{\left(e^{-x}+1\right) \log \left(e^{-x}+1\right)}{e^{x}+1} x^{s-1} d x\right. \\
& -\frac{1}{\left(1-2^{1-s}\right) \Gamma(s)} \int_{0}^{\infty} \frac{e^{-x} x^{s-1}}{e^{x}+1} d x,
\end{aligned}
$$




$$
\begin{gathered}
\zeta(s)+\frac{1}{\left(1-2^{1-s}\right) \Gamma(s)} \int_{0}^{\infty}\left[\frac{\left(e^{-x}+1\right) \log \left(e^{-x}+1\right)}{e^{x}+1}\right] x^{s-1} d x \\
-\frac{1}{\left(1-2^{1-s}\right) \Gamma(s)} \int_{0}^{\infty} \log \left(e^{-x}+1\right) e^{-x} x^{s-1} d x \\
=\frac{1}{1-2^{1-s}}-\frac{1}{\left(1-2^{1-s}\right) \Gamma(s)} \int_{0}^{\infty} \frac{e^{-x} x^{s-1}}{e^{x}+1} d x, \\
\zeta(s)+\frac{1}{\left(1-2^{1-s}\right) \Gamma(s)} \int_{0}^{\infty}\left[\frac{\left(e^{-x}+1\right) \log \left(e^{-x}+1\right)}{e^{x}+1}-\log \left(e^{-x}+1\right) e^{-x}\right] x^{s-1} d x \\
=\frac{1}{1-2^{1-s}}-\frac{1}{\left(1-2^{1-s}\right) \Gamma(s)} \int_{0}^{\infty} \frac{e^{-x} x^{s-1}}{e^{x}+1} d x, \\
\zeta(s)=\frac{1}{1-2^{1-s}}-\frac{1}{\left(1-2^{1-s}\right) \Gamma(s)} \int_{0}^{\infty} \frac{e^{-x} x^{s-1}}{e^{x}+1} d x, \\
\zeta(s)+\frac{1}{\left(1-2^{1-s}\right) \Gamma(s)} \int_{0}^{\infty} \frac{e^{-x} x^{s-1}}{e^{x}+1} d x=\frac{1}{1-2^{1-s}} .
\end{gathered}
$$

We calculate

$$
\int_{0}^{\infty} \frac{e^{-x} x^{s-1}}{e^{x}+1} d x=2^{-s} \Gamma(s)\left(\zeta(s)-\zeta\left(s, \frac{3}{2}\right)\right),
$$

for $\operatorname{Re}(s)>0$.

Substituting (2.8) in (2.7), we have

$$
\begin{gathered}
\zeta(s)+\frac{1}{\left(1-2^{1-s}\right) \Gamma(s)}\left\{2^{-s} \Gamma(s)\left[\zeta(s)-\zeta\left(s, \frac{3}{2}\right)\right]\right\}=\frac{1}{1-2^{1-s}} \\
\zeta(s)+\frac{\zeta(s)-\zeta\left(s, \frac{3}{2}\right)}{2^{s}-2}=\frac{1}{1-2^{1-s}} \\
\left(\frac{2^{s}-1}{2^{s}-2}\right) \zeta(s)=\frac{1}{1-2^{1-s}}+\frac{\zeta\left(s, \frac{3}{2}\right)}{2^{s}-2} \\
\left(\frac{2^{s}-1}{2^{s}-2}\right) \zeta(s)=\frac{2^{s}}{2^{s}-2}+\frac{\zeta\left(s, \frac{3}{2}\right)}{2^{s}-2} \\
\left(2^{s}-1\right) \zeta(s)=2^{s}+\zeta\left(s, \frac{3}{2}\right) \\
\zeta(s)=\frac{2^{s}}{2^{s}-1}+\frac{\zeta\left(s, \frac{3}{2}\right)}{2^{s}-1} . \square
\end{gathered}
$$

COROLLARY 1. Let $\zeta(s)=0$ and $\operatorname{Re}(s)>0$, then

$$
2^{s}+\zeta\left(s, \frac{3}{2}\right)=0
$$

holds.

Proof. If we assume that $\zeta(s)=0$, in Theorem 1, then 


$$
\frac{2^{s}}{2^{s}-1}+\frac{\zeta\left(s, \frac{3}{2}\right)}{2^{s}-1}=0 \Rightarrow 2^{s}+\zeta\left(s, \frac{3}{2}\right)=0 .
$$

The Theorem 2 can be rewritten as follows:

COROLLARY 1.a. For $\operatorname{Re}(s)>0$, all the roots of $\zeta(s)$ satisfies the equation

$$
2^{s}+\zeta\left(s, \frac{3}{2}\right)=0
$$

COROLLARY 2. For $\operatorname{Re}(s)>0$, then

$$
\left(2^{s}-1\right) \Gamma\left(\frac{1-s}{2}\right) \pi^{s-1 / 2} \zeta(1-s)=\Gamma\left(\frac{s}{2}\right)\left[2^{s}+\zeta\left(s, \frac{3}{2}\right)\right],
$$

and, for $0<\operatorname{Re}(s)<1$, then

$$
\left(2^{1-s}-1\right) \Gamma\left(\frac{s}{2}\right) \pi^{-s+1 / 2} \zeta(s)=\Gamma\left(\frac{1-s}{2}\right)\left[2^{1-s}+\zeta\left(1-s, \frac{3}{2}\right)\right],
$$

where $\Gamma(s)$ is the gamma function, $\zeta(s)$ is the Riemann zeta function and $\zeta(s, a)$ is the Hurwitz zeta function.

Proof. In [1] and [3, p. 136-144], we have

$$
\Gamma\left(\frac{s}{2}\right) \pi^{-s / 2} \zeta(s)=\Gamma\left(\frac{1-s}{2}\right) \pi^{-(1-s) / 2} \zeta(1-s) \Rightarrow \zeta(s)=\frac{\Gamma\left(\frac{1-s}{2}\right)}{\Gamma\left(\frac{s}{2}\right)} \pi^{s-1 / 2} \zeta(1-s),
$$

for all complex $s$, proved by Riemann (1859).

Substituting (2.12) in (2.3)

$\frac{\Gamma\left(\frac{1-s}{2}\right)}{\Gamma\left(\frac{s}{2}\right)} \pi^{s-1 / 2} \zeta(1-s)=\frac{2^{s}}{2^{s}-1}+\frac{\zeta\left(s, \frac{3}{2}\right)}{2^{s}-1} \Rightarrow\left(2^{s}-1\right) \Gamma\left(\frac{1-s}{2}\right) \pi^{s-1 / 2} \zeta(1-s)=\Gamma\left(\frac{s}{2}\right)\left[2^{s}+\zeta\left(s, \frac{3}{2}\right)\right]$.

Let $1-s \rightarrow s$ in $(2.13)$

$$
\left(2^{1-s}-1\right) \Gamma\left(\frac{s}{2}\right) \pi^{-s+1 / 2} \zeta(s)=\Gamma\left(\frac{1-s}{2}\right)\left[2^{1-s}+\zeta\left(1-s, \frac{3}{2}\right)\right] . \square
$$

THEOREM 2. Let $\operatorname{Re}(s)>1$, then

$$
\zeta(\mathrm{s})=\frac{2^{\mathrm{s}}}{2^{\mathrm{s}-1}}+\frac{3^{1-\mathrm{s}}}{\left(2-2^{1-\mathrm{s}}\right)(\mathrm{s}-1)}+\frac{2^{\mathrm{s}-1}}{3^{\mathrm{s}}\left(2^{\mathrm{s}}-1\right)}+\frac{2}{2^{\mathrm{s}}-1} \int_{0}^{\infty} \frac{\sin \left[\mathrm{s} \tan ^{-1}\left(\frac{2 \mathrm{t}}{3}\right)\right]}{\left[\left(\frac{3}{2}\right)^{2}+\mathrm{t}^{2}\right]^{\mathrm{s} / 2}} \frac{\mathrm{dt}}{\mathrm{e}^{2 \pi \mathrm{t}}-1},
$$

where $\zeta(s)$ is the Riemann zeta function.

Proof. In [4], we encounter the Abel-Plana formula for Hurwitz zeta function, that is,

$$
\zeta(\mathrm{s}, \alpha)=\sum_{\mathrm{n}=0}^{\infty} \frac{1}{(\mathrm{n}+\alpha)^{\mathrm{s}}}=\frac{\alpha^{1-\mathrm{s}}}{\mathrm{s}-1}+\frac{1}{2 \alpha^{\mathrm{s}}}+2 \int_{0}^{\infty} \frac{\sin \left(\mathrm{stan}^{-1} \frac{\mathrm{t}}{\alpha}\right)}{\left(\alpha^{2}+\mathrm{t}^{2}\right)^{\mathrm{s} / 2}} \frac{\mathrm{dt}}{\mathrm{e}^{2 \pi \mathrm{t}}-1} .
$$

Let $\alpha=\frac{3}{2}$ in $(2.15)$

$$
\zeta\left(\mathrm{s}, \frac{3}{2}\right)=\frac{3^{1-\mathrm{s}}}{2^{1-\mathrm{s}}(\mathrm{s}-1)}+\frac{2^{\mathrm{s}-1}}{3^{\mathrm{s}}}+2 \int_{0}^{\infty} \frac{\sin \left[\mathrm{stan}^{-1}\left(\frac{2 \mathrm{t}}{3}\right)\right]}{\left[\left(\frac{3}{2}\right)^{2}+\mathrm{t}^{2}\right]^{\mathrm{s} / 2}} \frac{\mathrm{dt}}{\mathrm{e}^{2 \pi \mathrm{t}}-1} .
$$

Substituting (2.16) in (2.3), we complete the proof. 
THEOREM 3. Let $\operatorname{Re}(s)>1$, then

$$
\zeta(s)=\frac{2^{s}}{2^{s}-1}+\frac{1}{(s-1)\left(2^{s}-1\right)}+\frac{1}{2^{s}-1} \sum_{n=0}^{\infty} \frac{(-1)^{n}}{n !} \gamma_{n}\left(\frac{3}{2}\right)(s-1)^{n},
$$

where $\zeta(s)$ is the Riemann zeta function and $\gamma_{n}(\alpha)$ are the Stieltjes constants.

Proof. In [5], we find the Laurent series expansion of the Hurwitz zeta function:

$$
\zeta(s, \alpha)=\frac{1}{s-1}+\sum_{n=0}^{\infty} \frac{(-1)^{n}}{n !} \gamma_{n}(\alpha)(s-1)^{n} .
$$

Let $\alpha=\frac{3}{2}$ in $(2.18)$

$$
\zeta\left(\mathrm{s}, \frac{3}{2}\right)=\frac{1}{\mathrm{~s}-1}+\sum_{\mathrm{n}=0}^{\infty} \frac{(-1)^{\mathrm{n}}}{\mathrm{n} !} \gamma_{\mathrm{n}}\left(\frac{3}{2}\right)(\mathrm{s}-1)^{\mathrm{n}} .
$$

Substituting (2.19) in (2.3), we complete the proof.

\section{REFERENCES}

[1] Sondow, Jonathan and Weisstein, Eric W., Riemann Zeta Function, from MathWorld--A Wolfram Web Resource, http://mathworld.wolfram.com/RiemannZetaFunction.html, available in May 06, 2013.

[2] Edwards, Harold M., Riemann's Zeta Function, Dover, 2001.

[3] Riemann, Bernhard, Bernhard Riemann's Gesammelte Mathematische Werke und Wissenschaftlicher Nachlass, "Ueber die Anzahl der Primzahlen unter einer gegebenen Grösse", Leipzig, 1876, Teubner.

[4] https://en.wikipedia.org/wiki/Abel-Plana_formula, available in May 07, 2013.

[5] https://en.wikipedia.org/wiki/Stieltjes_constants, available in May 07, 2013. 